\title{
RECENT ADVANCES IN PLANT PROTEOMICS TOWARDS STRESS ADAPTATION AND CROP IMPROVEMENT
}

\author{
REUBEN-KALU. J. I ${ }^{* 1} \&$ EKE-OKORO. O. N $^{2}$ \\ ${ }^{I}$ Center for Plant Molecular Biology and Biotechnology, Tamil Nadu Agricultural University, Coimbatore, India \\ ${ }^{2}$ National Root Crops Research Institute Umudike, Umuahia Abia State, Nigeria
}

\begin{abstract}
Biotic and abiotic stress conditions affect plant growth negatively resulting to low yield in crop production. Plant cells use combination of proteins to adjust their metabolism, growth and physiology as a function of the changes in their developmental stages and response to the environmental stress conditions around them. In this article, we look into the current advances in plant proteomics with emphasis on some case studies pointing out the applications of proteomics techniques for more knowledge on various response-mechanisms of plants to biotic and abiotic stress conditions at protein level towards crop improvement. For a detailed study of the plant proteome, more than one technique is required, hence the development of advanced quantitative and comparative proteomics techniques to improve the study and analysis of total proteomes and sub-proteomes via mass spectrometry-based proteomics coupled with sub-cellular fractionation protocols and the use of isobaric tags for relative and absolute quantification method, which have unraveled new opportunities to explain various biological processes taking place in plant cells especially in investigating biotic and abiotic stress responses. KEYWORDS: Abiotic Stress, Biotic Stress, Plant Proteome \& Advanced Proteomics Techniques
\end{abstract}

Received: May 18, 2020; Accepted: Jun 08, 2020; Published: Jul 06, 2020; Paper Id.: IJASRAUG20209

\section{INTRODUCTION}

Proteomics is the study or complete analysis of the entire complements of proteins found in a cell. It involves the identification, quantification, interactions and functions of proteins in a living cell. Proteomics analysis is one of the best procedures used in characterizing large sets of proteins in a given sample, including the functional analysis of translated regions of plant genome to unravel their biological processes and obtain detailed information about the mechanisms used by plants in response to stress conditions. In past, it was thought that proteins can be easily analyzed in a single experiment, but today scientists have come to understand that proteins as molecular entities are complex in nature and difficult to be completely analyzed in one experiment. The mass spectrometry technique used in the analysis of complex protein samples can result in up to several thousands of peptide spectra which will not be easily analyzed using any standard biochemical technique (Millar and Taylor 2014). Therefore, understanding the various mechanisms of adjustments and adaptation applied by crop plants during stress conditions are very important in order to get significant results in genomics-based breeding of crop plants for stress tolerance and high yield.

The transfixed nature of plants makes them to be continually exposed to adverse environmental conditions which lead to low production. To survive in any unfavourable environment, plants devise various adaptive mechanisms including biochemical and physiological traits (Komatsu and Hossain 2017). Currently, there has been great progress in the comprehension of how plants respond to environmental conditions, even at protein level. The 
present decade has recorded great achievements in the field of proteomics, especially in phase chromatography or two dimensional gel electrophoresis (2-DE), tandem and non-tandem mass spectrometry (MS), isobaric tags for relative and absolute quantification labeling technique (iTRAQ), X-ray crystallography and nuclear magnetic resonance. Therefore, the progression from gel-based proteomics to short-gun proteomics and mass spectrometry-based proteomics have been implemented, which have provided new platforms to be used in constructing proteome atlases for all plant species (Cheol et al., 2019). Proteome approaches are powerful tools which can assist in the investigation of comprehensive protein expression profiles in specific biological responses (Wang et al., 2018).

In the present review, we discuss current advances in plant proteomics studies via high-throughput proteomics analysis, mass spectrometry and use of advanced bioinformatics tools towards crop improvement. The availability of genomic information for various plant species, development of advanced bioinformatics tools and advanced analytical instruments make proteomics an important approach to give more insight into major signaling and biochemical pathways underlying plant life cycle, their interaction with the environment, and their responses to biotic and abiotic stress conditions (Yates and III 2019). Therefore, proteomics studies have progressed from simple identification of individual proteins to comparative and quantitative protein profiling, peptide mass fingerprinting, post-translational modification (PTM) analysis of proteins, sub-cellular localization, quantification of protein complexes, analysis of protein signaling pathways, and protein-protein interactions.

\section{PROTEOMICS ANALYSIS USING TWO-DIMENSIONAL GEL ELECTROPHORESIS}

Proteomics analysis based on two-dimensional gel electrophoresis is one of the methods commonly used to analyze proteomes and identify differentially accumulated proteins among various mutant populations of crop plants. With the use of two-dimensional gel electrophoresis, individual proteins contained in a sample can be identified. It is also useful when studying relatively abundant proteins in a given sample. However, 2-DE has some technical limitations which include: non-amenability to high throughput processing of many samples at the same time, detection of only the most abundant proteins in the sample, and requirement of expertise in generation of consistent results, which therefore makes it difficult to identify some lesion mimic mutants involving various proteins at the whole proteome level. Taking rice cultivars for example, among mutant population of japonica rice cultivars, only few numbers of differential proteins have been reported between the lesion mimic mutants and the wild type using 2-DE. Thus, about eighteen (18) differential proteins was recorded by Kim et al., (2008), thirty seven (37) was recorded by Tsunezuka et al., (2005), thirty three (33) was recorded by Jung et al., (2006) while one hundred and fifty nine (159) was recorded by Kang et al., (2007), which points out that the technical limitations have affected the advancement of proteomics research on lesion mimic mutants.

\section{HIGH-THROUGHPUT PROTEOMICS ANALYSIS}

High-throughput proteomics analysis can be carried out using iTRAQ-based method, which is an important tool employed in large-scale studies and comprehensive identification of drought responsive proteins in plants (Hu et al., 2015, Benešová et al., 2012, Gong et al., 2015, Dong et al., 2014). iTRAQ is a forward proteomics technique that provides a gel-free shortgun quantitative analysis where complex protein mixtures can be identified with high accuracy. It uses isobaric reagents to label tryptic peptides and monitor relative changes in protein and peptide mass tolerance abundance and can analyze several protein samples in a single experiment (Wu and Wang 2016). Protein peptides can also be analyzed via high pressure liquid chromatography (HPLC) which separates peptides on the basis of hydrophobicity. HPLC can be directly 
coupled to a time-flight mass spectrometer using the ionization of electrospray and the peptides coming from the column can be identified via tandem mass spectrometry.

It is expedient to evaluate the molecular changes taking place at protein level when studying the response of plants to stress conditions such as drought stress, cold stress, salinity stress, flooding etc since proteins are involved in stress response of crop plants. Proteomics studies are important when analyzing the relationships between changes at protein level and stress tolerance in plants (Wu et al., 2017), as new findings into the mechanisms used by plants in response to stress conditions at protein level can be obtained (Lei et al., 2015).

\section{PROTEOMICS ANALYSIS USING ITRAQ METHOD IN COMBINATION WITH LC-MS/MS}

iTRAQ coupled to liquid chromatography-quadruple mass spectrometry (LC-MS/MS) is another efficient proteomics approach for fast identification and accurate quantification of complex protein mixtures (Evans et al., 2012) and it is currently being used for quantitative and comparative analysis of plant proteomes to study the various environmental stress conditions affecting crop plants (Zhang et al., 2016, Li et al., 2015, Xie et al., 2016). iTRAQ combined with LC-MS/MS is widely used because of its high resolution and accurate protein expression profiles.

The availability of bioinformatics resources has made it possible to easily analyze and obtain data more accurately (Luo and Zhao 2012). Therefore, iTRAQ combined with LC-MS/MS technique has been widely used in studying abiotic and biotic stress responses in plants (Chen, et al., 2016, Wang et al., 2015).

\section{PROTEOMICS, AS A TOOL FOR STUDYING PLANT RESPONSE TO BIOTIC STRESS}

In the natural environment, plants are constantly affected by various biotic stress conditions which threaten their development, survival and growth (Zipfel and Oldroyd 2017). In response to these biotic stress conditions, plants develop some molecular response mechanisms which help them to perceive, adjust and adapt to the environmental conditions. The presence and role of normal proteins and stress-induced proteins observed in plants in response to biotic stress condition is very important due to the fact that: proteins are involved in the formation of various new phenotypes of plants through the regulation of physiological characteristics used in response to changes in the environment; also proteins are main executors of plant cellular functions and play vital roles in maintenance of the homeostasis functions within the cell. However, the various functions of proteins in plant cells are dependent upon their ability to specifically interact with other protein molecules since they do not function as individual molecules hence, they use coordinated and interlinked mechanisms to respond to biotic stress conditions in the environment (Yang et al., 2014).

Mass spectrometry-based proteomics has provided scientists with great ability to study plant responses to biotic stress conditions more precisely than it was possible years ago. Mass spectrometry technique is a useful tool applied in proteomics analysis of biotic stress conditions in plants, because it is a very sensitive technique and requires little amount of protein during the analysis. Also, a protein population can be prepared from a biological sample and the individual polypeptides separated with the aid of mass spectrometry-based proteomics approach. Therefore, proteomics study has evolved from being an isolated area of science to an extensive scientific area employed for biological research which can be useful in explaining various biological functions in living cells. Several studies have successfully used recent proteomics techniques as a discovery tool to unravel the adaptive mechanisms used by plant in response to biotic stress. There is also increasing results which indicate that post-translational modifications of proteins directly participating in plant immune activity during biotic stress. Hence, having deeper knowledge on cellular proteins, sub-cellular localization 
of proteins and post-translational modifications of proteins are very important so as to get comprehensive insights on the response of plants during biotic stress conditions (Liu et al., 2019) as the data obtained will help in proffering solutions towards crop improvement.

From researches carried out, numerous reports have been given on plant responses to biotic stress based on genetic, proteomic and genomic information obtained, thus research on genetic control and resistance mechanisms to biotic stress should result to the development of new crop cultivars with resistance traits to biotic stress conditions (Wang et al., 2019).The function of cellular protein does not depend only on its molecular structure but also on the sub-cellular localization and post-translational modifications (Kosova et al., 2018). The function of protein in plant cells during biotic stress is mainly linked to sub-cellular localization because different cell components perform different biochemical and physiological activities such as redox reactions and $\mathrm{pH}$ conditions. And most of the biological processes taking place in plant cells especially during biotic stress involve alterations in protein sub-cellular localization and transfer of mitochondrial proteins during cell death (Lundberg and Borner 2019).

\section{TOLERANCE MECHANISM IN PLANTS IN RESPONSE TO BIOTIC STRESS THROUGH PROTEIN REGULATION}

One of the tolerance mechanisms applied by plants in response to biotic stress is protein regulation. Therefore, it is important to know the proteins or proteomes that are involved in the biological process during protein regulation as the plants encounter biotic stress conditions. Scientists have spent several years analyzing how to advance proteome coverage in living cells, depending majorly on mass spectrometry-based techniques and bioinformatics analysis (Baerenfaller et al., 2008). Whole-cell proteome is made up of more than ten thousand different proteins, showing the importance for extensive research on whole-cell proteome (Millar and Taylor 2014, Wilhelm et al., 2014). However, after several years of expanding the metrical coverage of proteome, about 50\% coverage has successfully been achieved in Arabidopsis (Baerenfaller et al., 2008).

\section{PROTEOMICS ANALYSIS OF ABIOTIC STRESS RESPONSES IN PLANTS}

One of the major constraints for production of crop plants in specific areas globally is abiotic stress, and this is because of the rapid changes in the environmental conditions which are likely to override the adaptive potentials of crop plants. Therefore, it is a serious concern for food security especially in developing countries and should be given conscious efforts using advanced proteomics and genome-based breeding methods so that areas that are prone to abiotic stresses can have improved crop production.

The burden of abiotic stress conditions on plants such as metal toxicity, flood stress, cold stress, drought stress, salinity stress, heat stress, water stress etc affect the way plants grow and perform various functions which in turn affect crop productivity. Proteomics approach and techniques have been successfully used to study the metabolic processes that occur during these abiotic stress conditions in various plants via two dimensional gel electrophoresis coupled with mass spectrometry and iTRAQ coupled with (LC-MS/MS) analysis (Liang et al., 2006).

The recent advances in plant proteomics studies have contributed in illuminating on the complex relationship existing between stress tolerance in plants and crop productivity, which has enabled the development of novel breeding strategies that will lead to positive increase in environmental performance and crop production. The proteomics of leaf photosynthesis and senescence can be used to study crop productivity since leaf photosynthesis is the major source of plant 
biomass which influences crop productivity. Analyzing leaf photosynthesis using proteomics approach is very important when studying the measures for improvement of crop productivity since the presence of chlorophyll in leaves and chlorophyll metabolism are crucial for light harvesting by plants and also important for energy transfer during photosynthetic processes (Chu et al., 2015).

\section{CASE STUDIES}

Proteomics Analyses of Drought-Stress Tolerance Mechanisms in Maize

Drought stress is the most challenging abiotic stress which affects plant development. It causes serious threat to production of maize globally (Raos et al., 2016; Lei et al., 2015; Miao et al., 2017). Plants respond to drought stress using three main levels of adaptive mechanisms which are physiological, biochemical and molecular mechanisms (Zhu, 2016; Wang and Komatsu 2016). To have a clearer knowledge on the determinants of drought tolerance in maize, Zenda et al., (2018) recorded the analysis of two contrast inbred lines of maize (drought-tolerant variety and drought-sensitive variety) using proteomics approach. The two maize varieties were exposure to drought stress condition at the seedling stage for seven days and the molecular changes at protein level were evaluated using the iTRAQ method. It was recorded that, out of the total 721 differentially abundant proteins observed, 5 sets were drought responsive while 13 sets of the proteins were observed to be specific to one of the drought-tolerant inbred lines.

\section{IDENTIFICATION OF PROTEINS LINKED TO LESION MIMIC MUTANT IN RICE VIA PROTEOMICS ANALYSIS}

Yang et al., (2019) used iTRAQ-based strategy to identify novel rice lesion mimic mutant designated as E40 from a mutant population of japonica rice cultivar called Hitomebore. It was recorded that the proteins were analyzed to actually understand the altered pathways which caused cell death between the E40 mutant rice variety and the wild type, and the biological processes involved in formation of the lesions. The proteomics analysis identified a total of 2,722 proteins, and 233 proteins were observed to be differentially expressed, having diverse biological processes.

\section{PROTEOMICS ANALYSIS OF COLD STRESS RESPONSE IN JOJOBA}

Jojoba (Simmondsia chinensis) is a semi-arid and oil-producing crop mainly grown in tropical areas. Cold stress is one of the major abiotic stress conditions that affect the production of Jojoba in many temperate regions. Quantitative proteomics analysis was carried out with the leaves of Jojoba using iTRAQ-based technique to ascertain the mechanisms by which the crop responds to cold stress condition. The result revealed differentially accumulated proteins existing between the coldtreated plants and the control used in the experiment Gao et al., (2019).

When high-resolution LC-MS/MS was also employed to detect and quantify the amount of proteins present in Jojoba leaves during cold stress, it was found out that cold stress affected the leaf membrane integrity. The analysis also revealed that jojoba leaves respond to cold stress by reducing the content of leaf-chlorophyll, stopping photosynthesis, enhancing the relative oxygen species scavenging activities and adjusting the protein yield by regulating the abundance of various stress-response related proteins Gao et al., (2019).

The results obtained support the accuracy and reliability of iTRAQ and LC-MS/MS technology in investigating plant response to different abiotic stress conditions and also provide important data which will aid in understanding plant response to cold stress in tropical regions. 


\section{PROTEOMICS ANALYSIS OF CASTOR SEEDS IN RESPONSE TO COLD STRESS}

Cold stress is one of the major abiotic stresses that limit the growth of plants that are sensitive to low temperatures. Several plants of tropical origin are prone to cold damage when exposed to temperatures below $20{ }^{\circ} \mathrm{C}$ as they suffer intracellular freezing which causes damage to the protoplasmic structure of the affected plats. Castor bean is one of such seeds that are sensitive to cold stress in temperate regions and it has been reported that temperature below $20{ }^{\circ} \mathrm{C}$ drastically reduces the ability of the seeds to germinate (Jiang and Wen 2008).

Castor oil is an important raw material used for pharmaceutical and industrial purposes and a good source of ricinoleic acid and hydroxylated fatty acid (Scholz and Da Silva 2008). Castor beans can be grown in environments with drought and saline stress conditions where many other crops cannot survive and still produce great yield (Lima Da Silva et al., 2006). However, when planted during low temperatures castor seeds experience low germination ability as the initial imbibition of water affects the membrane integrity and blocks germination.

Wang et al., (2019) reported the early imbibition of castor seeds in response to cold stress through the application of quantitative proteomics analysis using iTRAQ and LC-MS/MS techniques. The results obtained showed that under cold stress, one hundred and twenty seven (127) differential abundance protein species were observed, where one hundred and nine (109) of the proteins were reported to be up-regulated while eighteen (18) were down-regulated when compared to control temperature conditions. Also, other cold-related proteins identified in the experiment such as methionine aminopeptidase has been confirmed in other plants using iTRAQ method as well. iTRAQ and LC-MS/MS techniques was also used in identifying some novel proteins that have not been previously observed to be associated with low temperature stress response and tolerance in castor seeds.

\section{ASSESSMENT OF THE ORGANIC AND INORGANIC CHEMICAL EFFECT ON SILVER NANOPARTICLES IN WHEAT VIA PROTEOMICS ANALYSIS}

Jhanzab et al., (2019) conducted proteomics analysis to identify the proteins that were affected in wheat samples in response to chemo-blended silver nanoparticles using Nano LC-MS/MS technique. In the results obtained glycolysisrelated proteins tend to reduce in response to chemo-blended silver nanoparticles, which suggests that glycolysis-related proteins play major role in regulating energy metabolism in wheat via glyceraldehyde -3-phosphate dehydrogenase and phosphoenol pyruvate carboxylase which was revealed through advanced proteomics technique: Nano LC-MS/MS.

\section{COLOUR CHANGE PROFILES OF BRUNFELSIA ACUMINATA FLOWERS USING PROTEOMICS APPROACH}

Brunfelsia acuminata is a popular ornamental plant that has various colours on the petals, which is as a result of the fast change of colour after blooming, hence proteomics approach such as the two dimensional electrophoresis is employed as one of the major methods of studying the proteomics of the entire floral parts, which has also been widely used in the study of other organs found in flowers, including the gynoecium, petals and androecium (Li et al., 2016).

With the aid of proteomics technique, the reason for the petal colour change was analyzed using gas chromatography coupled to a time-of-flight mass tool (Li et al., 2019). From the proteomics analysis, 52 proteins were identified and classified into 8 functional groups and $6 \%$ of the proteins were observed to be related to anthocyanin metabolic pathway. However, the expression levels of all the anthocyanin proteins were recorded to be down-regulated 
while most of the proteins linked to the defense abiotic stress and leaf senescence were up-regulated. Therefore, as anthocyanin content decreases, the petal colour changes from deep purple to white.

\section{LIMITATIONS IN PROTEOMICS ANALYSIS}

Some limitations exist in proteomics analysis, such as analysis concerned with the number of genes that code for proteins, detailed amount of proteins observed during analysis and analysis of proteomics data. These complex analytical techniques cause variability in data collection which leads to some problems in obtaining accurate results. The data obtained from proteomics analysis may have some limitations at each phase of the analysis which may include: i. Preparation of protein sample, extraction of the proteins. ii. Peptide separation. iii. Mass spectrometry-based analysis and iv. Bioinformatics tools employed for the interpretation of data obtained. However, to minimize any variability in proteomics analysis, a detailed and unbiased test kit is expedient which will lead to obtaining high-confidence proteomics results (Liu et al., 2019).

Also, the data analysis of plant proteomes for samples containing large amount of proteins, such as soybean seeds is still a limiting factor in proteomics study (Min et al., 2019). Thus, for future proteomics studies, it is important to increase the dynamic coverage of both model and non-model seed proteome using recent advances in proteomics technologies to overcome the complexities and limitations in the proteomics analysis of various seeds of economic important crops (Min et al., 2019).

\section{CONCLUSIONS AND SUGGESTION}

Plant cells use combination of protein molecules to adjust their metabolism, growth and physiology as a function of the changes in their developmental stages. Plant cell communications within the sub-cellular compartments take place in plants as response-mechanism to biotic and abiotic stress conditions around their environment. Sequel to the readily available and detailed information on nucleotide sequences in protein databases and the success recorded so far on rapid protein identification, recent proteomics approaches have opened new concepts and methodologies used in analyzing the complex protein functions of plants and various crop species at different levels, which have significantly taken the proteome coverage of many plants and crop species to another level towards crop improvement.

Despite the significant advances over the years, some limitations still exist in plant proteomics analysis including the number of genes that code for proteins, total number of proteins observed in a complex protein sample and analysis of proteomics data. Therefore, proteomics studies need adequate dry laboratory, time, and carefulness in data mining so that even missing proteins can be properly identified in any proteome analysis. However, the recent improvements in plant proteomics offers new and promising ways to encourage the use of advanced proteomics techniques by plant scientists for crop improvement programmes.

\section{ACKNOWLEDGEMENT}

The authors are grateful to Dr. Geetha, S., the Director of Centre for Plant Breeding and Genetics, Tamil Nadu Agricultural University Coimbatore-3 India, for assigning the first author to write on this topic.

\section{REFERENCES}

1. Baerenfaller, K., Grossmann, J., Grobei, M. A., Hull, R., Hirsch-Hoffmann, M., Yalovsky, S., Zimmermann, P., Grossniklaus, U., Gruissem, W and Baginsky, S. (2008). Genome-scale proteomics reveals Arabidopsis thaliana gene models and proteome dynamics. Science. 320 (5878): 938-41. 
2. Benešová, M., Holá, D., Fischer, L., Jedelský, P. L., Hnili`cka, F., Wilhelmová, N., Rothová, O., Ko`cová, M., Procházková, D and Honnerová, J. (2012). The physiology and proteomics of drought tolerance in maize: Early stomatal closure as a cause of lower tolerance to short-term dehydration? PLoS ONE. 7, e38017.

3. Chen, T., Zhang, L., Shang, H., Liu, S., Peng, J., Gong, W., Shi, Y., Zhang, S., Li, J. and Gong, J. (2016). iTRAQ-Based Quantitative Proteomic Analysis of Cotton Roots and Leaves Reveals Pathways Associated with Salt Stress. PLoS ONE, 11, e0148487.

4. Cheol, W. M., Ravi, G., Ganesh, K. A., Randeep, R. and Sun, T. K. (2019). Concepts and strategies of soybean seed proteomics using the shotgun proteomics approach, Expert Review of Proteomics., 16 (9): 795-804.

5. Chu, P., Yan, G. X., Yang, Q., Zhai, L. N., Zhang, C., Zhang, F. Q., et al. (2015). iTRAQ-based quantitative proteomics analysis of Brassica napus leaves reveals pathways associated with chlorophyll deficiency. J. Proteomics 113: 110-126.

6. Dong, M., Gu, J., Zhang, L., Chen, P., Liu, T., Deng, J., Lu, H., Han, L. and Zhao, B. (2014). Comparative proteomics analysis of superior and inferior spikelets in hybrid rice during grain filling and response of inferior spikelets to drought stress using isobaric tags for relative and absolute quantification. J. Proteom., 109, 382-399.

7. Evans, C., Noirel, J., Ow, S.Y., Salim, M., Pereira-Medrano, A. G., Couto, N., Pandhal, J., Smith, D., Pham, T. K. and Karunakaran, E. (2012). An insight into iTRAQ: Where do we stand now? Anal. Bioanal. Chem. 404: 1011-1027.

8. Gao, F., Ma, P., Wu, Y., Zhou, Y. and Zhang, G. (2019). Quantitative proteomic analysis of the response to cold stress in jojoba, a tropical woody crop. Int. J. Mol. Sci., 20, 243.

9. Gong, F., Hu, X. and Wang, W. (2015). Proteomic analysis of crop plants under abiotic stress conditions: Where to focus our research? Front. Plant Sci., 6, 418.

10. Hu, X., Wu, L., Zhao, F., Zhang, D., Li, N., Zhu, G., Li, C and Wang, W. (2015). Phosphoproteomic analysis of the response of maize leaves to drought, heat and their combination stress. Front. Plant Sci., 6, 298.

11. Jhanzab, H., Razzaq, A., Bibi, Y., Yasmeen, F., Yamaguchi, H., Hitachi, K., Tsuchida, K. and Komatsu, S. (2019). Proteomic analysis of the effect of inorganic and organic chemicals on silver nanoparticles in wheat. Int. J. Mol. Sci. 20: 825.

12. Jiang, X. J and Wen, X. D. (2008). The effect of temperature on the germination rates in castor bean. Seed., 27: 67-69.

13. Jung, Y., Rakwal, R., Agrawal, G., Shibato, J., Kim, J., Lee, M., Choi, P., Jung, S., Kim, S and Koh, H. (2006). Differential expression of defense/stress-related marker proteins in leaves of a unique rice blast lesion mimic mutant (blm). J. Proteome Res., 5: 2586-2598.

14. Kang, S. G., Matin, M. N., Bae, H and Natarajan, S. (2007). Proteome analysis and characterization of phenotypes of lesion mimic mutant spotted leaf 6 in rice. Proteomics. 7: 2447-2458.

15. Kim, S., Kim, S., Kang, Y., Wang, Y., Kim, J., Yi, N., Kim, J., Rakwal, R and Hj Kang, K. (2008). Proteomics analysis of rice lesion mimic mutant (spill) reveals tightly localized Probenazole-Induced protein (PBZ1) in cells undergoing programmed cell death. J. Proteome Res., 7: 1750-1760.

16. Komatsu, S and Hossain, Z. (2017). Plant Proteomic Research. Int. J. Mol. Sci. 18, 88.

17. Kosova, K., Vitamvas, P., Urban, M. O., Prasil, I. T and Renaut, J. (2018). Plant abiotic stress proteomics: the major factors determining alterations in cellular proteome. Front Plant Sci., 9:122.

18. Lei, L., Shi, J., Chen, J., Zhang, M., Sun, S., Xie, S., Li, X., Zeng, B., Peng, L. and Hauck, A. (2015). Ribosome profiling reveals dynamic translational landscape in maize seedlings under drought stress. Plant J., 84, 1206-1218. 
19. Li, M., Sun, Y., Lu, X., Debnath, B., Mitra, S. and Qiu, D. (2019). Proteomics reveal the profiles of color change in Brunfelsia acuminata flowers. Int. J. Mol. Sci., 20: 2000.

20. Li, W., Zhao, F., Fang, W., Xie, D., Hou, J., Yang, X., Zhao, Y., Tang, Z., Nie, L and Lv, S. (2015). Identification of early salt stress responsive proteins in seedling roots of upland cotton (Gossypium hirsutum L.) employing iTRAQ-based proteomic technique. Front. Plant Sci. 6; 732.

21. Li, X., Jackson, A., Xie, M.; Wu, D., Tsai, W.C. and Zhang, S. (2016). Proteomic insights into floral biology. BBA Proteins Proteomics., 1864, 1050-1060.

22. Liang, X. A., Luo, M. and Guo, B. Z. (2006). Resistance Mechanisms to Aspergillus falvus infectin and aflatoxin contamination in peanut (Arachis hypogaea). Plant Pathology Journal 5: 115-124.

23. Lima Da Silva, N., Maciel, M. Batistella, C. and Filho, R. (2006). Optimization of biodiesel production from castor oil. Appl. Biochem. Biotechnol., 130, 405-414.

24. Liu, Y., Song, L., Kefu Liu, Sheng, W., Luqi, H. and Lanping G. (2019). Proteomics: a powerful tool to study plant responses to biotic stress. Plant methods. 15: 135.

25. Lundberg, E and Borner, G. H. H. (2019). Spatial proteomics: a powerful discovery tool for cell biology. Nat Rev Mol Cell Biol., 20(5):285-302.

26. Luo, R. and Zhao, H. (2012). Protein quantitation using iTRAQ: Review on the sources of variations and analysis of nonrandom missingness. Stat. Interface, 5: 99-107.

27. Miao, Z., Han, Z., Zhang, T., Chen, S., and Ma, C. A. (2017). Systems approach to spatio-temporal understanding of the drought stress response in maize. Sci. Rep., 7, 1-14.

28. Millar, A. H. and Taylor, N. L. (2014). Subcellular proteomics-where cell biology meets protein chemistry. Front Plant Sci., $5: 55$.

29. Min, C. W., Gupta, R., Agrawal, G. K., Rakwal, R and T. Kim, S. T. (2019). Concepts and strategies of soybean seed proteomics using the shotgun proteomics approach. Expert Rev proteomics., (9): 795-804.

30. Raos, G. J., N., Reddy, J. N., Variar, M. and Mahender, A. (2016). Molecular breeding to improve plant breeding to improve plant resistance to abiotic stresses. Adv. Plant Breed. Strateg., 2, 283-326.

31. Scholz, V and Da Silva, J. N. (2008). Prospects and risks of the use of castor oil as a fuel. Biomass Bioenergy. 32, 95-100.

32. Tsunezuka, H., Fujiwara, M. and Kawasaki, T. (2005). Shimamoto, K. Proteome analysis of programmed cell death and defense signaling using the rice lesion mimic mutant cdr2. Mol. Plant-Microbe Interact. 18: 52.

33. Wang, B., Hajano, J. U. D., Ren, Y., Lu, C. and Wang, X. (2015). iTRAQ-based quantitative proteomics analysis of rice leaves infected by Rice stripe virus reveals several proteins involved in symptom formation. Virol. J., 12: 99.

34. Wang, D., Eraslan, B., Wieland, T., Hallstrom, B., Hopf, T., Zolg, D. P., Zecha, J., Asplund, A., Li, L. H., Meng, C., Frejno, M., Schmidt, T., Schnatbaum, K., Wilhelm, M., Ponten, F., Uhlen, M., Gagneur, J., Hahne, H and Kuster, B. (2019). A deep proteome and transcriptome abundance atlas of 29 healthy human tissues. Mol Syst Biol., 15(2): e8503.

35. Wang, X and Komatsu, S. (2016). Plant sub-cellular proteomics: Application for exploring optimal cell function in soybean. J. Proteom., 143, 45-56. 
36. Wang, X., Li, M., Liu, X., Zhang, L., Duan, Q. and Zhang, J. (2019). Quantitative proteomic analysis of castor (Ricinus communis L.) seeds during early imbibition provided novel insights into cold stress response. Int. J. Mol. Sci., 20: 355.

37. Wang, Z. Q., Zhou, X., Dong, L., Guo, J., Chen, Y., Zhang, Y., Wu, L. and Xu, M. (2018). iTRAQ-based analysis of the Arabidopsis proteome reveals insights into the potential mechanisms of anthocyanin accumulation regulation in response to phosphate deficiency. J. Proteomics., 184, 39-53.

38. Wilhelm, M., Schlegl, J., Hahne, H., Gholami, A. M., Lieberenz, M., Savitski, M. M, Ziegler, E., Butzmann, L., Gessulat, S., Marx, H., Mathieson, T., Lemeer, S., Schnatbaum, K., Reimer, U., Wenschuh, H., Mollenhauer, M., SlottaHuspenina, J., Boese, J. H., Bantscheff, M., Gerstmair, A., Faerber, F. and Kuster B. (2014). Mass-spectrometry-based draft of the human proteome. Nature., 509 (7502): 582-7.

39. $W u$, S., Ning, F., Zhang, $Q$., Wu, X. and Wang, W. (2017). Enhancing omics research of crop responses to drought under field conditions. Front. Plant Sci. 2017, 8, 1-5.

40. Wu, X and Wang, W. (2016). Increasing confidence of proteomics data regarding the identification of stress-responsive proteins in crop plants. Front. Plant Sci. 7, 702.

41. Xie, H., Yang, D. H., Yao, H., Bai, G., Zhang, Y. H. and Xiao, B. G. (2016). iTRAQ-based quantitative proteomic analysis reveals proteomic changes in leaves of cultivated tobacco (Nicotiana tabacum) in response to drought stress. Biochem. Biophys. Res. Commun. 469: 768-775.

42. Yang, L., Jiang, T., Fountain, J. C., Scully, B. T., Lee, R. D., Kemerait, R. C., Chen, S. and Guo, B. (2014). Protein profiles reveal diverse responsive signaling pathways in kernels of two maize inbred lines with contrasting drought sensitivity. Int. J. Mol. Sci., 15, 18892-18918.

43. Yang, X., Meng, W., Zhao, M., Zhang, A., Liu, W., Xu, Z., Wang, Y. and Ma, J. (2019). Proteomics analysis to identify proteins and pathways associated with the novel lesion mimic mutant E40 in rice using iTRAQ-based strategy. Int. J. Mol. Sci., 20: 1294.

44. Yates J. and III J. R. (2019). Recent technical advances in proteomics. F1000 Faculty Rev., 8::351.

45. Zenda, T., Liu, S., Wang, X., Jin, H.; Liu, G.; and Duan, H. (2018). Comparative proteomic and physiological analyses of two divergent maize inbred lines provide more insights into drought-stress tolerance mechanisms. Int. J. Mol. Sci., 19, 3225.

46. Zhang, W., Zhang, H., Ning, L., Li, B. and Bao, M. (2016). Quantitative proteomic analysis provides novel insights into cold stress responses in petunia seedlings. Front. Plant Sci. 7: 137.

47. Zhu, J. K. (2016). Abiotic stress signaling and responses in plants. Cell. 167, 313-324.

48. Zipfel, C and Oldroyd, G. E. (2017). Plant signalling in symbiosis and immunity. Nature. 543(7645): 328-36. 